\title{
Retinal Embolization Syndrome as a Perioperative Complication of Surgical Restoration of Internal Carotid Artery - Case Report
}

\author{
Joanna Dolar-Szczasny ${ }^{1}$, Anna Święch-Zubilewicz ${ }^{1}$, Tomasz Żarnowski ${ }^{2}$ and Jerzy \\ Mackiewicz $^{1}$ \\ ${ }^{1}$ Department of Retina and Vitreal Surgery of Medical University in Lublin, Lublin, Poland \\ ${ }^{2}$ Department of Glaucoma Diagnosis and Treatment of Medical University in Lublin, Lublin, Poland
}

Correspondence should be addressed to: Joanna Dolar-Szczasny; joannaszczasny@op.pl

Received date: 5 May 2014; Accepted date: 22 September 2014; Published date: 28 August 2015

Academic Editor: Arijana Lovrenčić-Huzjan

Copyright (C) 2015. Joanna Dolar-Szczasny, Anna Święch-Zubilewicz, Tomasz Żarnowski and Jerzy Mackiewicz. Distributed under Creative Commons CC-BY 4.0

\begin{abstract}
Embolization of cholesterol material from atherosclerotic plaques lining the walls of major arteries causes different types of cholesterol embolization syndromes (CES), depending on the organs being involved. CES is most frequently found after cardiovascular procedures and less common after carotid surgery. We describe a case of a 65 -year old heavily smoker male patient of Caucasian origin with high grade symptomatic carotid stenosis. Duplex Doppler examination and angio-CT showed more than $90 \%$ unstable plaque in the right carotid artery. Clinically, he presented three transient ischemic attacks (TIA) in the past 2 years, the last one two months before surgical procedure. Carotid endarterectomy (CEA) was performed in local anaesthesia without using shunt and primary closure. Postoperative period was complicated in the second day by important visual deterioration in the right eye and the patient was referred to the Ophthalmological Department. In the indirect ophthalmoscopy of the right eye, a whitening of the superior retinal artery area and many cholesterol plaques was noted. Additionally, performed OCT and fluorescein angiography confirmed a diagnosis of retinal embolization syndrome. The present case report shows that there is a rare possibility of embolic retinal arteries occlusion (RAO) due to CES as a complication of the surgical restoration of internal carotid artery.
\end{abstract}

Keywords: cholesterol embolization syndrome (CES), carotid endarterectomy (CEA), retinal arteries occlusion (RAO)

\section{Introduction}

Cholesterol embolization syndrome (CES) refers to the embolization of cholesterol crystals from ruptured atherosclerotic plaques lining the walls of proximally located arteries. The most common physical findings are cutaneous manifestations, and the most common procedures having high rate of CES are cardiovascular procedures [1].

Cite this Article as: Joanna Dolar-Szczasny, Anna Święch-Zubilewicz, Tomasz Żarnowski and Jerzy Mackiewicz (2015), "Retinal Embolization Syndrome as a Perioperative Complication of Surgical Restoration of Internal Carotid Artery - Case Report,” International Journal of Case Reports in Medicine, Vol. 2015 (2015), 
The clinical expressions of the disease vary according to the occluded area. The skin of the lower limbs, abdominal organs and kidneys are the most frequently involved organs with expressions of acrocyanosis, livedo reticularis and kidney failure, although symptoms may also appear in the brain and skeletal muscles. Retinal embolization after carotid endarterectomy (CEA) is extremely rare. Only one case of such complication has been published so far and it concerned a central retinal artery occlusion (RAO) [2].

We present a case of retinal embolization syndrome as a postoperative complication occurring after a surgical restoration of internal carotid artery.

\section{Case Presentation}

We describe a case of a 65 -year old heavily smoker male patient with stable coronary artery disease (CAD), hypertension and high grade symptomatic carotid stenosis. The preoperative work-up included neurological examination, duplex ultrasonography and contrast arteriography. Duplex Doppler examination and angio-CT showed more than $90 \%$ unstable plaque having heterogeneous morphology in the right carotid artery. The patient had a combination of aspirin and statin treatment. The total cholesterol level was $180 \mathrm{mg} / \mathrm{dL}$ (HDL - $50 \mathrm{mg} / \mathrm{dL}, \mathrm{LDL}-110$ $\mathrm{mg} / \mathrm{dL}$ ) on the day of admission.

The left carotid artery and both vertebral arteries were patent without reverse flow or any significant haemodynamic changes. Clinically, he presented three transient ischemic attacks (TIA) in the past 2 years, the last one two months before surgical procedure. CEA was performed in locoregional anaesthesia with combined cervical plexus block without using shunt.

The skin incision was made parallel to the anterior border of the right sternocleidomastoid muscle. Exposure and clamping of the common carotid artery (CCA), external carotid artery (ECA) and ICA were made in the usual way. Next systemic heparinization was used. A right - angled buldog clamp controlled the ICA beyond the distal end of the atheromatous plaque. The incision was made parallel to the axis of the ECA. The atheroma was separated and detached.

The arteriotomy was repaired with a 7-0 prolene suture and patch angioplasty.

The duration of ICA clamping was 25 minutes. An intraluminal shunt was not used in this procedure.

A bolus of heparin (5000 U) was administered prior to ICA clamping, and protamine was administered at the end of surgery.

Blood pressure was stable in a range $\pm 20 \%$ of the preoperative level throughout the procedure.

The postoperative period was complicated in the second day by important visual deterioration in the right eye and the patient was referred to the Ophthalmological Department. Control ultrasonography screening detected any new morphologic alterations in the extracranial part of carotid end vertebral arteries. Echocardiography examination did not reveal an embolic source or any valve lesions. No hematological and ECG abnormalities were identified.

On the initial ophthalmic evaluation best, the corrected visual acuity in a right eye was 0.2 and in a left eye - 0.9. Intraocular pressure in both eyes was within normal limits. Anterior segment examination revealed slight lens opacity in both eyes. In the indirect ophthalmoscopy of the right eye, multiple cholesterol plaques in vessels of superior hemispheral region and a whitening of superior retinal artery area were noted. 


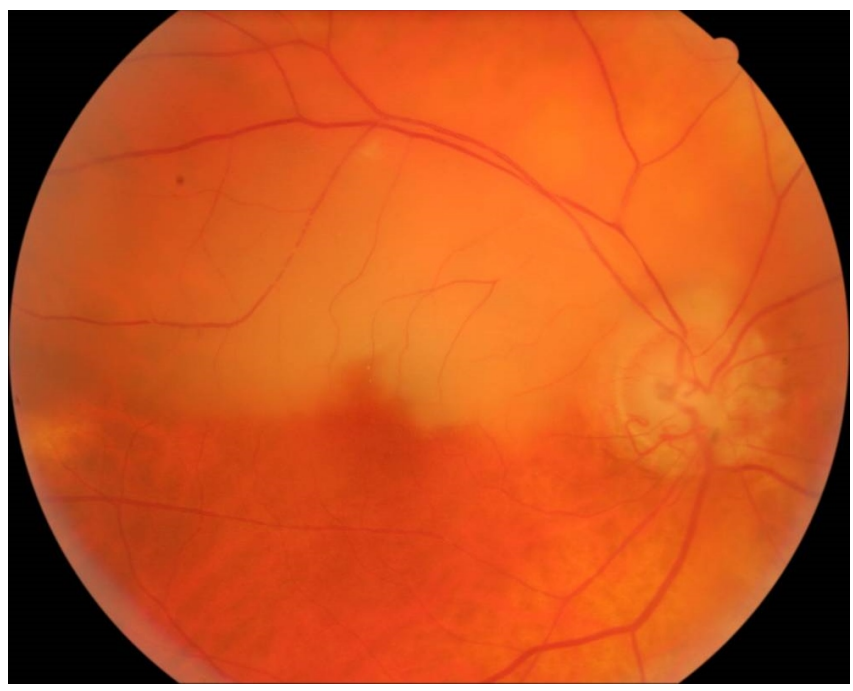

Figure 1. Colour Fundus Photograph of the Right Eye Presenting Many Sites of Embolic Material Prominently Visible within the Retinal Arterioles in the Superior Branch of Retinal Artery. There is a Broad Area of Retinal Edema in the Region Supplied by the Occluded Arteries.

The left eye had all signs of a second stage of hypertonic angiopathy.

We performed optical coherence tomography (OCT) examination using Zeiss Cirrus HDOCT and fluorescein angiography using
Heidelberg Retina Angiograph 2 device. In the OCT scans of the right eye, thickening of the inner retinal layers - typical for retinal artery occlusion - in the clinically visible abnormality area was present.

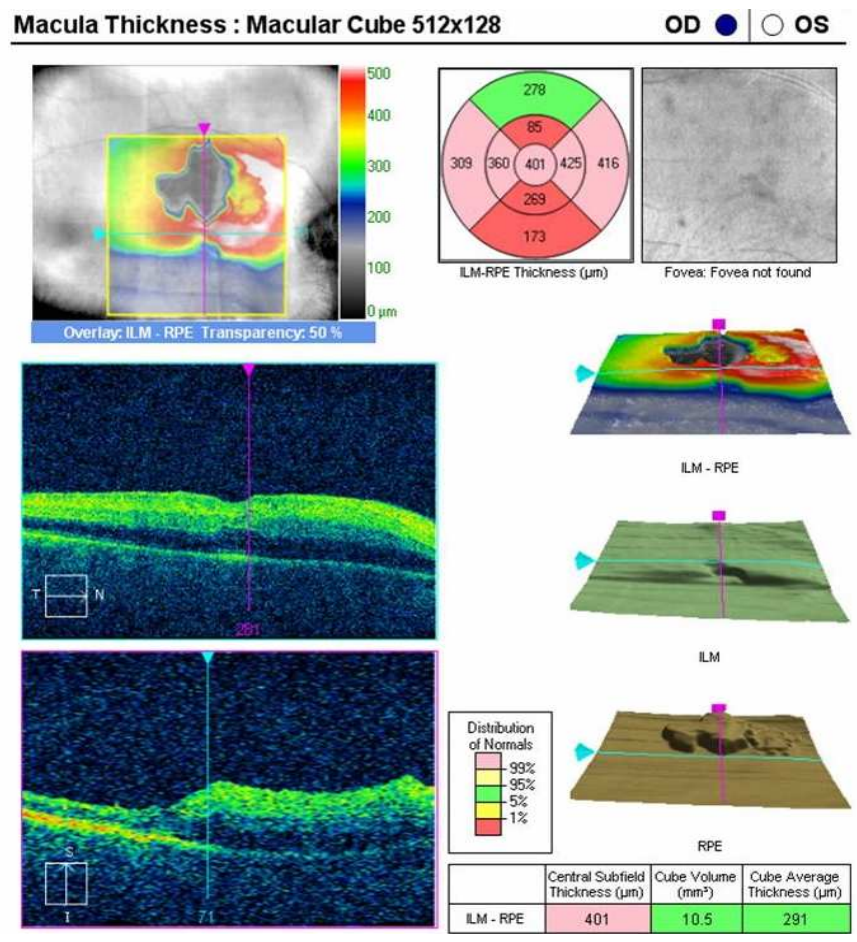

Figure 2. OCT Image of the Right Eye with Thickening of the inner Retinal Layers in the Superior Part of the Macular Region 
Fluorescein angiography revealed delayed filling of many arterioles from the superior branch of the retinal artery and the concomitant oedema surrounding the superior branch of the central artery in the right eye.

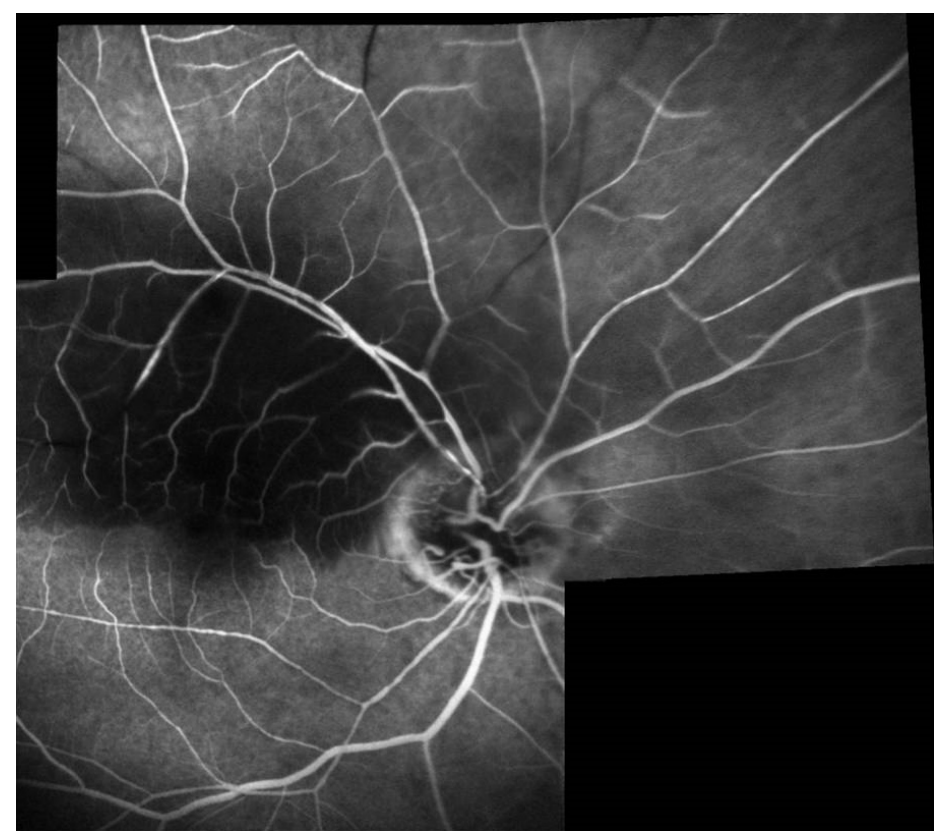

Figure 3. Fluorescein Angiography Composite Image Showing Delayed Filling of Affected Retinal Vessels with Some of them Showing Segments without Perfusion. Infarcted Retina is Oedematous with Blocked Background Fluorescence.

The presented additional examinations confirmed the diagnosis of retinal embolization syndrome in the right eye. We decided to implement standard treatment for retinal artery occlusion disorders.

Despite the immediate lowering intraocular pressure therapy (anterior chamber paracentesis + timolol eye-drops topically and 2 tablets of acetazolamide administered orally) during follow-up, after 1, 3 and 6 months we did not observe any distinct improvement in visual acuity.

\section{Discussion}

The two main clinical manifestations of CES are peripheral cutaneous involvement and acute renal insufficiency. The precise clinical syndrome depends on the location of the source of embolism. Since the abdominal aorta is the most common location of atherosclerosis, the kidneys, visceral organs, and lower extremities are commonly involved. Atheromas of other arteries, such as the ascending aorta or the carotid, may result in emboli, producing neurologic, retinal, or cardiac symptoms. The risk factors for CES include advanced age, smoking, hypertension, diabetes mellitus, hyperlipidemia, and peripheral vascular disease. CES is seen following invasive vascular procedures as in our patient, thrombolytic therapy, or anticoagulants, but may occur spontaneously as well [1].

CES has been observed after both surgical CEA and percutaneous carotid stenting. Most perioperative complications are ischemic cerebral lesions and they are due to CES or hypoperfusion from carotid clamping, being a part of the surgical procedure. On the contrary, the events in the later postoperative period are related to thromboembolism from the newly debrided arterial walls or dissection of an intimal flap [3].

Carotid stenting is emerging as an alternative procedure to carotid endarterectomy in patients with significant carotid artery 
stenosis. Nevertheless, still numerous microemboli can be shed during carotid angioplasty [4]. The risk of periprocedural ischemic strokes due to embolism after carotid stenting is about $5 \%$. This risk is nowadays considerably lowered due to the use of embolism protection devices [5-7].

The most common cause of RAO is embolism. In stable conditions (without any invasive procedures), the carotid artery and the heart are the most common sources. Carotid artery disease can cause RAO by three mechanisms: embolism, hemodynamically and serotonin induced arterial spasm. The major source of emboli is plaques in the carotid arteries, like in the present case, and much less frequently stenosis. On the contrary, during and after cardiovascular procedures, embolic material originates from other arteries [8-10].

Atheromatous plaque embolism may occur at the time of CEA procedure when plaque material dislodges and embolizes to intracranial vessels, like central retinal artery or branches of retinal artery. It may also occur at a later time as it happened in our case.

In the present case, clinical evaluation suggests that cholesterol plaques moved during CEA are the main cause of retinal embolization. It is uncommon according to the fact that most CESs happen after endovascular procedures and are attributable to endovascular catheter usage.

\section{Conclusions}

The present case report shows that there is a rare possibility of RAO due to CES as a complication of the surgical restoration of ICA. Detached intravascular embolic material in the reopened artery is probable cause of this complication.

\section{References}

1. Saric, M. \& Kronzon, I. (2011). "Cholesterol Embolization Syndrome," Current Opinion in Cardiology, 26 (6) 472-479.

2. Nucci, C., Martelli, E., Appolloni, A. \& Palma, S. (1997). "A Case of Central Retinal Artery
Occlusion after Carotid Endoarterectomy," Eye (London, England), 11 (Pt 5) 755-757.

3. Sila, C. A. (1998). "Neurologic Complications of Vascular Surgery," Neurologic Clincs, 16 (1) 9-20.

4. Rapp, J. H., Pan, X. M., Yu, B., Swanson, R. A., Higashida, R. T., Simpson, P. \& Saloner, D. (2003). "Cerebral Ischemia and Infarction from Atheroemboli <100 Microm in Size," Stroke, 34 (8) 1976-1980.

5. Zahn, R., Mark, B., Niedermaier, N., Zeymer, U., Limbourg, P., Ischinger, T., Haerten, K., Hauptmann, K. E., Leitner, E. R., Kasper, W., Tebbe, U. \& Senges, J. (2004). “Embolic Protection Devices for Carotid Artery Stenting: Better Results than Stenting without Protection?," European Heart Journal, 25 (17) 1550-1558.

6. Roubin, G. S., Iyer, S., Halkin, A., Vitek, J. \& Brennan, C. (2006). "Realizing the Potential of Carotid Artery Stenting: Proposed Paradigms for Patient Selection and Procedural Technique," Circulation, 113 (16) 2021-2030.

7. Kastrup, A., Groschel, K., Krapf, H., Brehm, B. R., Dichgans, J. \& Schulz, J. B. (2003). "Early Outcome of Carotid Angioplasty and Stenting with and without Cerebral Protection Devices: A Systematic Review of the Literature," Stroke, 34 (3) 813-819.

8. Blanco, V. R., Moris, C., Barriales, V. \& Gonzalez, C. (2000). "Retinal Cholesterol Emboli during Diagnostic Cardiac Catheterization," Catheterization and Cardiovascular Interventions, 51 (3) 323-325.

9. Bucci, F. A. Jr., Dimitsopulos, T. M. \& Krohel, G. B. (1989). "Branch Retinal Artery Occlusion Secondary to Percutaneous Transluminal Coronary Angioplasty," British Journal of Ophthalmology, 73 (4) 309-310.

10. Stefansson, E., Coin, J. T., Lewis, W. R. 3rd., Belkin, R. N., Behar, V. S., Morris, J. J. Jr. \& Anderson, W. B. (1985). "Central Retinal Artery Occlusion during Cardiac Catheterization," American Journal of Ophthalmology, 99 (5) 586-589. 